ANNALES

POLONICI MATHEMATICI

$82.3(2003)$

\title{
On the fiber product preserving gauge bundle functors on vector bundles
}

\author{
by WŁodzimierz M. MikUlski (Kraków)
}

\begin{abstract}
We present a complete description of all fiber product preserving gauge bundle functors $F$ on the category $\mathcal{V} \mathcal{B}_{m}$ of vector bundles with $m$-dimensional bases and vector bundle maps with local diffeomorphisms as base maps. Some corollaries of this result are presented.
\end{abstract}

Introduction. Modern differential geometry has clarified that product preserving bundle functors on the category $\mathcal{M} f$ of manifolds and maps play very important roles. To such bundle functors one can lift some geometric structures as vector fields, forms, connections, etc. To define such lifts the only important property is the product preservation. Such functors have been classified by means of Weil algebras [5].

Research quite similar to that on manifolds has been done on fibered manifolds. A wide class of bundle functors on the category $\mathcal{F M}_{m}$ of fibered manifolds with $m$-dimensional bases and fiber preserving maps with local diffeomorphisms as base maps is the class of fiber product preserving functors. Such functors have been classified in [6], and studied in [1]-[4], [8].

In turn research similar to that on fibered manifolds has been done on vector bundles. A wide class of (gauge) bundle functors on the category $\mathcal{V} \mathcal{B}_{m}$ of vector bundles with $m$-dimensional bases and vector bundle maps with local diffeomorphisms as base maps is the class of fiber product preserving functors. For example the $r$-jet prolongation functor plays an important role in the theory of higher order connections, Lagrangians, differential equations, etc. Below, we present many examples of such functors. Some of them are well known. It seems natural and useful to classify all such functors. The purpose of the present paper is to describe all fiber product preserving gauge bundle functors on $\mathcal{V B}_{m}$.

2000 Mathematics Subject Classification: 58A05, 58A20.

Key words and phrases: (fiber product preserving) gauge bundle functors, natural transformations, jets, Weil algebras. 
We first recall the following definitions (see e.g. [5]).

Let $F: \mathcal{V} \mathcal{B}_{m} \rightarrow \mathcal{F M}$ be a covariant functor into the category $\mathcal{F M}$ of fibered manifolds and their fibered maps. Let $B_{\mathcal{V} \mathcal{B}_{m}}: \mathcal{V} \mathcal{B}_{m} \rightarrow \mathcal{M f}$ and $B_{\mathcal{F} \mathcal{M}}: \mathcal{F} \mathcal{M} \rightarrow \mathcal{M} f$ be the respective base functors.

A gauge bundle functor on $\mathcal{V B}_{m}$ is a functor $F$ as above satisfying:

(i) (Base preservation) $B_{\mathcal{F} \mathcal{M}} \circ F=B_{\mathcal{V} \mathcal{B}_{m}}$. Hence the induced projections form a functor transformation $\pi: F \rightarrow B \mathcal{V} \mathcal{B}_{m}$.

(ii) (Localization) For every inclusion of an open vector subbundle $i_{E \mid U}$ : $E \mid U \rightarrow E, F(E \mid U)$ is the restriction $\pi^{-1}(U)$ of $\pi: F E \rightarrow B_{\mathcal{V} \mathcal{B}_{m}}(E)$ to $U$ and $F i_{E \mid U}$ is the inclusion $\pi^{-1}(U) \rightarrow F E$.

(iii) (Regularity) $F$ transforms smoothly parametrized systems of $\mathcal{V B}_{m^{-}}$ morphisms into smoothly parametrized systems of $\mathcal{F} \mathcal{M}$-morphisms.

A gauge bundle functor $F: \mathcal{V} \mathcal{B}_{m} \rightarrow \mathcal{F M}$ is of finite order $r$ if from $j_{x}^{r} f=j_{x}^{r} g$ it follows that $F_{x} f=F_{x} g$ for any $\mathcal{V B}_{m}$-objects $E_{1} \rightarrow M_{1}$, $E_{2} \rightarrow M_{2}$, any $\mathcal{V} \mathcal{B}_{m}$-maps $f, g: E_{1} \rightarrow E_{2}$ and any $x \in M_{1}$.

Given two gauge bundle functors $F_{1}, F_{2}$ on $\mathcal{V B}_{m}$, by a natural transformation $\mu: F_{1} \rightarrow F_{2}$ we mean a system of base preserving fibered maps $\mu: F_{1} E \rightarrow F_{2} E$ for every vector bundle $E$ satisfying $F_{2} f \circ \mu=\mu \circ F_{1} f$ for every $\mathcal{V} \mathcal{B}_{m}$-morphism $f: E \rightarrow G$.

A gauge bundle functor $F$ on $\mathcal{V} \mathcal{B}_{m}$ is fiber product preserving if for any fiber product projections

$$
E_{1} \stackrel{\mathrm{pr}_{1}}{\longleftarrow} E_{1} \times_{M} E_{2} \stackrel{\mathrm{pr}_{2}}{\longrightarrow} E_{2}
$$

in the category $\mathcal{V} \mathcal{B}_{m}$,

$$
F E_{1} \stackrel{F \mathrm{pr}_{1}}{\longleftarrow} F\left(E_{1} \times_{M} E_{2}\right) \stackrel{F \mathrm{pr}_{2}}{\longrightarrow} F E_{2}
$$

are fiber product projections in the category $\mathcal{F} \mathcal{M}$. In other words, we have $F\left(E_{1} \times_{M} E_{2}\right)=F\left(E_{1}\right) \times_{M} F\left(E_{2}\right)$ modulo the corestriction of $\left(F \mathrm{pr}_{1}, F \mathrm{pr}_{2}\right)$.

The most important example of a fiber product preserving gauge bundle functor is the $r$-jet prolongation functor $J^{r}: \mathcal{V} \mathcal{B}_{m} \rightarrow \mathcal{F M}$, where for a $\mathcal{V B}_{m}$-object $p: E \rightarrow M$ we have $J^{r} E=\left\{j_{x}^{r} \sigma \mid \sigma\right.$ is a local section of $E$, $x \in M\}$ and for a $\mathcal{V} \mathcal{B}_{m}$-map $f: E_{1} \rightarrow E_{2}$ covering $f: M_{1} \rightarrow M_{2}$ we have $J^{r} f: J^{r} E_{1} \rightarrow J^{r} E_{2}$, where $J^{r} f\left(j_{x}^{r} \sigma\right)=j_{\underline{f}(x)}^{r}\left(f \circ \sigma \circ \underline{f}^{-1}\right)$ for $j_{x}^{r} \sigma \in J^{r} E_{1}$.

Another example is the so-called vertical $r$-jet prolongation functor $J_{v}^{r}$ : $\mathcal{V} \mathcal{B}_{m} \rightarrow \mathcal{F M}$, where for a $\mathcal{V} \mathcal{B}_{m}$-object $p: E \rightarrow M$ we have $J_{v}^{r} E=\left\{j_{x}^{r} \gamma \mid\right.$ $\gamma$ is a local map $\left.M \rightarrow E_{x}, x \in M\right\}$ and for a $\mathcal{V} \mathcal{B}_{m}$-map $f: E_{1} \rightarrow E_{2}$ covering $\underline{f}: M_{1} \rightarrow M_{2}$ we have $J_{v}^{r} f: J_{v}^{r} E_{1} \rightarrow J_{v}^{r} E_{2}$, where $J_{v}^{r} f\left(j_{x}^{r} \gamma\right)=$ $j_{\underline{f(x)}}^{r}\left(f \circ \bar{\gamma} \circ \underline{f}^{-1}\right)$ for $j_{x}^{r} \gamma \in J_{v}^{r} E_{1}$.

Still another example is the vertical Weil functor $V^{A}: \mathcal{V B}_{m} \rightarrow \mathcal{F M}$ corresponding to a Weil algebra $A$, where for a $\mathcal{V B}_{m^{-}}$object $p: E \rightarrow M$ 
we have $V^{A} E=\bigcup_{x \in M} T^{A}\left(E_{x}\right)$ and for a $\mathcal{V} \mathcal{B}_{m}$-map $f: E_{1} \rightarrow E_{2}$ we have $V^{A} f=\bigcup_{x \in M_{1}} T^{A}\left(f_{x}\right): V^{A} E_{1} \rightarrow V^{A} E_{2}$. The functor $V^{A} E$ is equivalent to $E \otimes A$.

One more example is the following vector bundle modification $T_{f l}^{(r)}$ : $\mathcal{V} \mathcal{B}_{m} \rightarrow \mathcal{F} \mathcal{M}$ of the vector $r$-tangent bundle $T^{(r)}$ over manifolds. For a vector bundle $p: E \rightarrow M$ from $\mathcal{V} \mathcal{B}_{m}$ we have a vector bundle $T_{f l}^{r *} E=$ $J_{f l}^{r}(E, \mathbb{R})_{0}=\left\{j_{x}^{r} \gamma \mid \gamma: E \rightarrow \mathbb{R}\right.$ is fiber linear, $\left.\gamma_{x}=0, x \in M\right\}$ over $M$, where $\gamma_{x}$ is the restriction of $\gamma$ to the fiber $E_{x}$ of $E$ over $x \in M$. Let $T_{f l}^{(r)} E=\left(T_{f l}^{r *} E\right)^{*}$ be the dual vector bundle. Every $\mathcal{V B}_{m}$-map $f: E \rightarrow \bar{E}$ covering $\underline{f}: M \rightarrow \bar{M}$ induces a $\mathcal{V} \mathcal{B}_{m}$-map $T_{f l}^{(r)} f: T_{f l}^{(r)} E \rightarrow T_{f l}^{(r)} \bar{E}$ covering $\underline{f}$, where $\left\langle T_{f l}^{(r)} f(\omega), j_{f(x)}^{r} \gamma\right\rangle=\left\langle\omega, j_{x}^{r}(\gamma \circ f)\right\rangle$ for $\omega \in\left(T_{f l}^{(r)}\right)_{x} E$, and $\gamma: \bar{E} \rightarrow \mathbb{R}$ is fiber linear with $\gamma_{\underline{f}(x)}=0, x \in M$.

The fiber product $F_{1} \times_{B_{\mathcal{V} \mathcal{B}_{m}}} F_{2}: \mathcal{V B}_{m} \rightarrow \mathcal{F} \mathcal{M}$ of fiber product preserving gauge bundle functors $F_{1}, F_{2}: \mathcal{V} \mathcal{B}_{m} \rightarrow \mathcal{F M}$ is again a fiber product preserving gauge bundle functor. We recall that $\left(F_{1} \times_{B_{\mathcal{V} \mathcal{B}_{m}}} F_{2}\right)(E)=$ $F_{1} E \times_{M} F_{2} E$ for any $\mathcal{V} \mathcal{B}_{m}$-object $E \rightarrow M$, and $\left(F_{1} \times_{B_{\mathcal{V} \mathcal{B}_{m}}} F_{2}\right)(f)\left(v_{1}, v_{2}\right)=$ $\left(F_{1} f\left(v_{1}\right), F_{2} f\left(v_{2}\right)\right)$ for any $\mathcal{V} \mathcal{B}_{m}$-map $f: E \rightarrow G$ and any $\left(v_{1}, v_{2}\right) \in F_{1} E \times_{M}$ $F_{2} E$.

The composition of fiber product preserving gauge bundle functors on $\mathcal{V} \mathcal{B}_{m}$ is again a fiber product preserving gauge bundle functor on $\mathcal{V B}_{m}$. (In Lemma 1 it will be proved that every fiber product preserving gauge bundle functor has values in $\mathcal{V B}_{m}$. So, the composition is possible.)

If $F: \mathcal{V} \mathcal{B}_{m} \rightarrow \mathcal{F M}$ is a fiber product preserving gauge bundle functor of order $r$ we define a new fiber product preserving gauge bundle functor $\left(F\left(^{*}\right)\right)^{*}: \mathcal{V} \mathcal{B}_{m} \rightarrow \mathcal{F} \mathcal{M}$ by $E \mapsto\left(F E^{*}\right)^{*}$ and $f \mapsto\left(F f^{*}\right)^{*}$, where ()$^{*}$ denotes the dualization of $\mathcal{V} \mathcal{B}_{m}$-objects and $\mathcal{V} \mathcal{B}_{m}$-maps.

The first main result in this paper is that all fiber product preserving gauge bundle functors $F$ on $\mathcal{V} \mathcal{B}_{m}$ of finite order $r$ are in bijection with socalled admissible triples, i.e. triples $(V, H, t)$ where $V$ is a finite-dimensional vector space over $\mathbb{R}, H: G_{m}^{r} \rightarrow \mathrm{GL}(V)$ is a smooth group homomorphism from $G_{m}^{r}=\operatorname{inv} J_{0}^{r}\left(\mathbb{R}^{m}, \mathbb{R}^{m}\right)_{0}$ into $\mathrm{GL}(V)$ and $t: \mathcal{D}_{m}^{r} \rightarrow \operatorname{gl}(V)$ is a $G_{m^{-}}^{r}$ equivariant unital associative algebra homomorphism from $\mathcal{D}_{m}^{r}=J_{0}^{r}\left(\mathbb{R}^{m}, \mathbb{R}\right)$ into $\mathrm{gl}(V)$.

The second main result is that natural transformations between two fiber product preserving gauge bundle functors on $\mathcal{V B}_{m}$ of order $r$ are in bijection with morphisms between the corresponding admissible triples.

The third main result is that any fiber product preserving gauge bundle functor on $\mathcal{V} \mathcal{B}_{m}$ is of finite order.

As corollaries of the above results we describe explicitly all natural endomorphisms $J^{r} \rightarrow J^{r}, J_{v}^{r} \rightarrow J_{v}^{r}$ and $V^{A} \rightarrow V^{A}$ for any Weil algebra $A$. 
All manifolds are assumed to be finite-dimensional. All manifolds and maps are assumed to be smooth, i.e. of class $\mathcal{C}^{\infty}$.

\section{Fiber product preserving gauge bundle functors on $\mathcal{V} \mathcal{B}_{m}$ cor- responding to admissible triples}

DEFINITION 1. An admissible triple of order $r$ and dimension $m$ is a triple $(V, H, t)$, where $V$ is a finite-dimensional vector space over $\mathbb{R}, H$ : $G_{m}^{r} \rightarrow \mathrm{GL}(V)$ is a smooth group homomorphism from the Lie group $G_{m}^{r}=$ inv $J_{0}^{r}\left(\mathbb{R}^{m}, \mathbb{R}^{m}\right)_{0}$ of invertible $r$-jets at $0 \in \mathbb{R}^{m}$ of diffeomorphisms $\mathbb{R}^{m} \rightarrow$ $\mathbb{R}^{m}$ preserving 0 into the group $\mathrm{GL}(V)$ of linear isomorphisms of $V$, and $t: \mathcal{D}_{m}^{r} \rightarrow \operatorname{gl}(V)$ is a $G_{m}^{r}$-equivariant unital algebra homomorphism from the unital algebra $\mathcal{D}_{m}^{r}=J_{0}^{r}\left(\mathbb{R}^{m}, \mathbb{R}\right)$ of $r$-jets at $0 \in \mathbb{R}^{m}$ of maps $\mathbb{R}^{m} \rightarrow \mathbb{R}$ into the unital associative algebra $\mathrm{gl}(V)$ of linear endomorphisms of $V$.

We recall that $G_{m}^{r}$ acts on $\mathcal{D}_{m}^{r}$ by $j_{0}^{r} \varphi \cdot j_{0}^{r} \gamma=j_{0}^{r}\left(\gamma \circ \varphi^{-1}\right)$ for $j_{0}^{r} \varphi \in G_{m}^{r}$, $j_{0}^{r} \gamma \in \mathcal{D}_{m}^{r}$. This action will be denoted by $H_{m}^{r}$. We also recall that $G_{m}^{r}$ acts on $\operatorname{gl}(V)$ by $\xi . A=H(\xi) \circ A \circ H\left(\xi^{-1}\right)$ for $\xi \in G_{m}^{r}, A \in \operatorname{gl}(V)$. These actions are by unital algebra isomorphisms.

Let $(V, H, t)$ be an admissible triple of order $r$ and dimension $m$. We are going to construct a fiber product preserving gauge bundle functor $T^{(V, H, t)}$ : $\mathcal{V} \mathcal{B}_{m} \rightarrow \mathcal{F M}$.

EXAMPLE 1. For a vector bundle $p: E \rightarrow M$ from $\mathcal{V B}_{m}$ we put

$$
T^{(V, H, t)} E=\bigcup_{x \in M} \operatorname{Hom}_{t_{x}}\left(J^{r} \mathcal{C}_{x}^{\infty, f l}(E), \widetilde{V}_{x} M\right) .
$$

Here $\widetilde{V}: \mathcal{M} f_{m} \rightarrow \mathcal{V B}$ is the natural vector bundle corresponding to the $G_{m}^{r}$ space $V$, i.e. $\widetilde{V} M=P^{r} M[V, H]$ (the associated bundle) for any $m$-manifold $M$ and $\widetilde{V} \varphi=P^{r} \varphi\left[\operatorname{id}_{V}\right]: \widetilde{V} M_{1} \rightarrow \widetilde{V} M_{2}$ for any embedding $\varphi: M_{1} \rightarrow M_{2}$ between $m$-manifolds, and $\operatorname{Hom}_{t_{x}}\left(J^{r} \mathcal{C}_{x}^{\infty, f l}(E), \widetilde{V}_{x} M\right)$ is the space of module homomorphisms over $t_{x}: J_{x}^{r}(M, \mathbb{R}) \rightarrow \operatorname{gl}\left(\widetilde{V}_{x} M\right)$ from the (free) $J_{x}^{r}(M, \mathbb{R})$ module $J^{r} \mathcal{C}_{x}^{\infty, f l}(E)$ of $r$-jets at $x \in M$ of germs at $x$ of fiber linear maps $E \rightarrow \mathbb{R}$ into the $\operatorname{gl}\left(\widetilde{V}_{x} M\right)$-module $\widetilde{V}_{x} M$, where $t_{x}: J_{x}^{r}(M, \mathbb{R}) \rightarrow \operatorname{gl}\left(\widetilde{V}_{x} M\right)$ is the unital algebra homomorphism induced by $t$ such that $t_{x}\left(j_{x}^{r} \gamma\right)=\widetilde{V}_{0} \varphi \circ$ $t\left(j_{0}^{r}(\gamma \circ \varphi)\right) \circ\left(\widetilde{V}_{0} \varphi\right)^{-1}$ for any $\gamma: M \rightarrow \mathbb{R}$ and any embedding $\varphi: \mathbb{R}^{m} \rightarrow M$ with $\varphi(0)=x$ ( $t_{x}$ is well defined because $t$ is $G_{m}^{r}$-equivariant).

Given a vector bundle trivialization $\left(x^{1} \circ p, \ldots, x^{m} \circ p, y^{1}, \ldots, y^{n}\right)$ : $E \mid U \rightarrow \mathbb{R}^{m} \times \mathbb{R}^{n}$ we have an induced fiber bundle trivialization $\left(\widetilde{x}^{1}, \ldots, \widetilde{x}^{m}\right.$, $\left.\widetilde{y}^{1}, \ldots, \widetilde{y}^{n}\right): T^{(V, H, t)} E \mid U \rightarrow \mathbb{R}^{m} \times V^{n}$ such that $\widetilde{x}^{i}(\Phi)=x^{i}\left(x_{0}\right) \in \mathbb{R}$ and $\widetilde{y}^{j}(\Phi)=\Phi\left(j_{x_{0}}^{r}\left(y^{i}\right)\right) \in \widetilde{V}_{x_{0}} M \cong V$ for any $\Phi \in \operatorname{Hom}_{t_{x_{0}}}\left(J^{r} \mathcal{C}_{x_{0}}^{\infty, f l}(E), \widetilde{V}_{x_{0}} M\right)$, $i=1, \ldots, m, j=1, \ldots, n$, where $V \cong \widetilde{V}_{x_{0}} M$ is given by $v \leftrightarrow \widetilde{V}\left(\left(x^{1}, \ldots, x^{m}\right)^{-1}\right.$ 
$\left.\circ \tau_{\left(x^{i}\left(x_{0}\right)\right)}\right)(v)$ for $v \in V$, and $\tau_{y}: \mathbb{R}^{m} \rightarrow \mathbb{R}^{m}$ is the translation by $y \in \mathbb{R}^{m}$. Then $T^{(V, H, t)} E$ with the obvious projection is a fiber bundle over $M$.

Every $\mathcal{V B}_{m}$-map $f: E_{1} \rightarrow E_{2}$ covering $\underline{f}: M_{1} \rightarrow M_{2}$ induces a fibered map $T^{(V, H, t)} E_{1} \rightarrow T^{(V, H, t)} E_{2}$ covering $\underline{f}$ such that

$$
T^{(V, H, t)} f(\Phi)\left(j_{\underline{f}(x)}^{r} \xi\right)=\widetilde{V} \underline{f} \circ \Phi\left(j_{x}^{r}(\xi \circ f)\right)
$$

for any $\Phi \in \operatorname{Hom}_{t_{x}}\left(J^{r} \mathcal{C}_{x}^{\infty, f l}\left(E_{1}\right), \widetilde{V}_{x} M\right), x \in M_{1}$, and any fiber linear map $\xi: E_{2} \rightarrow \mathbb{R}$.

If in some vector bundle trivializations $f(x, y)=\left(\underline{f}(x), \sum_{j} f^{j}(x) y_{j}\right)$ for $x \in \mathbb{R}^{m}, y=\left(y_{1}, \ldots, y_{n}\right) \in \mathbb{R}^{n}, f^{j}=\left(f_{l}^{j}\right): \mathbb{R}^{m} \rightarrow \mathbb{R}^{k}, j=1, \ldots, n$, $l=1, \ldots, k$, then in the induced trivializations

$$
\begin{aligned}
T^{(V, H, t)} & f(x, v) \\
& =\left(\underline{f}(x),\left(\sum_{j} t\left(j_{0}^{r}\left(f_{l}^{j} \circ \underline{f}^{-1} \circ \tau_{\underline{f}(x)}\right)\right)\left(H\left(j_{0}^{r}\left(\tau_{-\underline{f}(x)} \circ \underline{f} \circ \tau_{x}\right)\right)\left(v_{j}\right)\right)\right)_{l=1}^{k}\right)
\end{aligned}
$$

for $x \in \mathbb{R}^{m}$ and $v=\left(v_{1}, \ldots, v_{n}\right) \in V^{n}$.

The correspondence $T^{(V, H, t)}: \mathcal{V} \mathcal{B}_{m} \rightarrow \mathcal{F M}$ is a fiber product preserving gauge bundle functor of order $r$ and it takes values in the category $\mathcal{V} \mathcal{B}_{m}$.

Definition 2. We call $T^{(V, H, t)}: \mathcal{V} \mathcal{B}_{m} \rightarrow \mathcal{F} \mathcal{M}$ the fiber product preserving gauge bundle functor corresponding to the admissible triple $(V, H, t)$.

\section{Admissible triples corresponding to fiber product preserving gauge bundle functors on $\mathcal{V} \mathcal{B}_{m}$}

Lemma 1. Let $F: \mathcal{V B}_{m} \rightarrow \mathcal{F} \mathcal{M}$ be a f.p.p.g.b. functor.

(a) Given a vector bundle $p: E \rightarrow M$ we have a canonical vector bundle structure on FE.

(b) Given a $\mathcal{V B}_{m}$-map $f: E_{1} \rightarrow E_{2}$ over $\underline{f}: M_{1} \rightarrow M_{2}$ the induced map $F f: F E_{1} \rightarrow F E_{2}$ is a vector bundle map over $\underline{f}$.

Proof. The fiber sum map $+^{E}: E \times_{M} E \rightarrow E$, the fiber scalar multiplication maps $\lambda_{t}^{E}: E \rightarrow E$ for $t \in \mathbb{R}$ and the zero map $0^{E}: E \rightarrow E$ are $\mathcal{V} \mathcal{B}_{m}$-maps and we can apply the functor $F$. We obtain $+{ }^{F E}:=F\left(+{ }^{E}\right)$ : $F E \times{ }_{M} F E \cong F\left(E \times_{M} E\right) \rightarrow F E, \lambda_{t}^{F E}:=F\left(\lambda_{t}^{E}\right): F E \rightarrow F E$ and $0^{F E}:=F\left(0^{E}\right): F E \rightarrow F E$. It is easily seen that $\left(F E,+{ }^{F E}, \lambda_{t}^{F E}, 0^{F E}\right)$ is a vector bundle structure on $F E$.

Let $F: \mathcal{V} \mathcal{B}_{m} \rightarrow \mathcal{F} \mathcal{M}$ be a f.p.p.g.b. functor of order $r$. We are going to construct an admissible triple $\left(V^{F}, H^{F}, t^{F}\right)$.

EXAMPLE 2. We put

$$
V^{F}:=F_{0}\left(\mathbb{R}^{m} \times \mathbb{R}\right)
$$


the fiber at $0 \in \mathbb{R}^{m}$ of the vector bundle $F\left(\mathbb{R}^{m} \times \mathbb{R}\right)$, where $\mathbb{R}^{m} \times \mathbb{R}$ is (of course) the trivial vector bundle over $\mathbb{R}^{m}$ with fiber $\mathbb{R}$. Then $V^{F}$ is a finite-dimensional vector space over $\mathbb{R}$.

We define $H^{F}: G_{m}^{r} \rightarrow \mathrm{GL}\left(V^{F}\right)$ by

$$
H^{F}(\xi)(v):=F_{0}\left(\varphi \times \operatorname{id}_{\mathbb{R}}\right)(v), \quad v \in V^{F}, \xi=j_{0}^{r} \varphi \in G_{m}^{r} .
$$

$H^{F}(\xi)(v)$ is well defined because $F$ is of order $r$. By the definition of $V^{F}$, $H^{F}(\xi) \in \mathrm{GL}\left(V^{F}\right)$. By the functoriality of $F, H^{F}$ is a group homomorphism. By the regularity of $F, H^{F}$ is smooth.

We define $t^{F}: \mathcal{D}_{m}^{r} \rightarrow \operatorname{gl}\left(V^{F}\right)$ by

$$
t^{F}(\eta)(v)=F_{0}(\widetilde{\gamma})(v), \quad v \in V^{F}, \eta=j_{0}^{r} \gamma \in \mathcal{D}_{m}^{r},
$$

where $\widetilde{\gamma}: \mathbb{R}^{m} \times \mathbb{R} \rightarrow \mathbb{R}^{m} \times \mathbb{R}$ is a $\mathcal{V} \mathcal{B}_{m^{-}}$map such that $\widetilde{\gamma}(x, y)=(x, \gamma(x) y)$ for $x \in \mathbb{R}^{m}, y \in \mathbb{R}$. Then $t^{F}(\eta)(v)$ is well defined because $F$ is of order $r$. By the definition of $V^{F}, t^{F}(\eta) \in \operatorname{gl}\left(V^{F}\right)$. By the functoriality of $F$ and the definitions of the actions one can verify in a standard (but long) way that $t^{F}$ is a $G_{m}^{r}$-equivariant unital algebra homomorphism.

Then $\left(V^{F}, H^{F}, t^{F}\right)$ is an admissible triple of order $r$ and dimension $m$.

Definition 3. We call $\left(V^{F}, H^{F}, t^{F}\right)$ the admissible triple corresponding to $F$.

\section{Admissible triples corresponding to some fiber product pre-} serving gauge bundle functors on $\mathcal{V B}_{m}$. In this section we present admissible triples corresponding to fiber product preserving gauge bundle functors on $\mathcal{V B}_{m}$ presented in the introduction. The results of this section will not be used to prove the main result.

FACT 1. The admissible triple corresponding to the $r$-jet prolongation gauge bundle functor $J^{r}: \mathcal{V} \mathcal{B}_{m} \rightarrow \mathcal{F} \mathcal{M}$ is $\left(\mathcal{D}_{m}^{r}, H_{m}^{r}, t_{m}^{r}\right)$, where $H_{m}^{r}: G_{m}^{r} \rightarrow$ $\operatorname{Aut}\left(\mathcal{D}_{m}^{r}\right)$ is defined after Definition 1 and $t_{m}^{r}: \mathcal{D}_{m}^{r} \rightarrow \operatorname{gl}\left(\mathcal{D}_{m}^{r}\right)$ is given by $t_{m}^{r}(\eta)(\varrho)=\eta \varrho$ for $\eta, \varrho \in \mathcal{D}_{m}^{r}$.

FACT 2. The admissible triple corresponding to the vertical $r$-jet prolongation gauge bundle functor $J_{v}^{r}: \mathcal{V} \mathcal{B}_{m} \rightarrow \mathcal{F} \mathcal{M}$ is $\left(\mathcal{D}_{m}^{r}, H_{m}^{r}, t_{m}^{r} \circ \varepsilon_{m}^{r}\right)$, where $H_{m}^{r}: G_{m}^{r} \rightarrow \operatorname{Aut}\left(\mathcal{D}_{m}^{r}\right)$ is defined after Definition $1, t_{m}^{r}: \mathcal{D}_{m}^{r} \rightarrow \operatorname{gl}\left(\mathcal{D}_{m}^{r}\right)$ is defined above and $\varepsilon_{m}^{r}: \mathcal{D}_{m}^{r} \rightarrow \mathbb{R} \subset \mathcal{D}_{m}^{r}$ is the algebra homomorphism.

FACT 3. The admissible triple corresponding to the vertical Weil gauge bundle functor $V^{A}: \mathcal{V B}_{m} \rightarrow \mathcal{F M}$ corresponding to a Weil algebra $A$ is $\left(A, \mathrm{id}_{A}, \varepsilon^{A}\right)$, where $\operatorname{id}_{A}: G_{m}^{r} \rightarrow\left\{\operatorname{id}_{A}\right\} \subset \mathrm{GL}(A)$ is the trivial group homomorphism and $\varepsilon^{A}: \mathcal{D}_{m}^{r} \rightarrow \operatorname{gl}(A)$ is given by $\varepsilon^{A}(\eta)(a)=\gamma(0)$ a for $\eta=j_{0}^{r} \gamma$ $\in \mathcal{D}_{m}^{r}, a \in A$.

The gauge bundle functors of Facts 1-3 are "almost restrictions" of fiber product preserving bundle functors on $\mathcal{F} \mathcal{M}_{m}$. In general, let $F: \mathcal{F} \mathcal{M}_{m} \rightarrow$ 
$\mathcal{F M}$ be a fiber product preserving bundle functor and $(A, H, t)$ be its corresponding triple in the sense of [6]. Then by "almost restriction" we have a fiber product preserving gauge bundle functor $\widetilde{F}: \mathcal{V} \mathcal{B}_{m} \rightarrow \mathcal{F M}$ given by $\widetilde{F} E=F E$ for any $\mathcal{V} \mathcal{B}_{m}$-object $E$ and $\widetilde{F} f=F f$ for any $\mathcal{V} \mathcal{B}_{m}$-map $f$.

FACT 4 . The admissible triple corresponding to $\widetilde{F}$ is $(\widetilde{A}, \widetilde{H}, \widetilde{t})$, where $\widetilde{A}$ is the vector space $A, \widetilde{H}: G_{m}^{r} \rightarrow \operatorname{GL}(\widetilde{A})$ is $H: G_{m}^{r} \rightarrow \operatorname{Aut}(A) \subset \operatorname{GL}(\widetilde{A})$ and $\widetilde{t}: \mathcal{D}_{m}^{r} \rightarrow \operatorname{gl}(\widetilde{A})$ is given by $\widetilde{t}(\eta)(a)=t(\eta)$ a for $a \in \widetilde{A}$ and $\eta \in \mathcal{D}_{m}^{r}$.

The gauge bundle functor $V^{A}$ of Fact 3 is a particular case of the following general construction. Let $\widetilde{V}: \mathcal{M} f_{m} \rightarrow \mathcal{V B}$ be the natural vector bundle corresponding to a group homomorphism $H: G_{m}^{r} \rightarrow \mathrm{GL}(V)$, i.e. $\widetilde{V} M=P^{r} M[V, H]$ (the associated bundle) for any $m$-manifold $M$ and $\widetilde{V} \varphi=P^{r} \varphi\left[\operatorname{id}_{V}\right]: \widetilde{V} M_{1} \rightarrow \widetilde{V} M_{2}$ for any embedding $\varphi: M_{1} \rightarrow M_{2}$ between $m$-manifolds. Let $\widetilde{\widetilde{V}}: \mathcal{V} \mathcal{B}_{m} \rightarrow \mathcal{V B}$ be the fiber product preserving gauge bundle functor such that $\widetilde{\widetilde{V}} E=E \otimes_{M} \widetilde{V} M$ for any $\mathcal{V B}_{m^{-}}$object $p: E \rightarrow M$ and $\widetilde{\widetilde{V}} f=f \otimes \widetilde{V} f: \widetilde{\widetilde{V}} E_{1} \rightarrow \widetilde{\widetilde{V}} E_{2}$ for any $\mathcal{V} \mathcal{B}_{m}$-map $f: E_{1} \rightarrow E_{2}$ covering $\underline{f}: M_{1} \rightarrow M_{2}$. (For $V=A$ and trivial $H: G_{m}^{r} \rightarrow\left\{\operatorname{id}_{V}\right\} \subset \mathrm{GL}(V)$ we obtain $V^{A}$.)

FACT 5. The admissible triple corresponding to the above $\widetilde{\widetilde{V}}: \mathcal{V} \mathcal{B}_{m} \rightarrow$ $\mathcal{F M}$ is $\left(V, H, \varepsilon^{V}\right)$, where $\varepsilon^{V}: \mathcal{D}_{m}^{r} \rightarrow \operatorname{gl}(V)$ is given by $\varepsilon^{V}(\eta)(v)=\gamma(0) v$ for $\eta=j_{0}^{r} \gamma \in \mathcal{D}_{m}^{r}, v \in V$.

FACT 6. The admissible triple $(V, H, t)$ of order $r$ corresponding to $T_{f l}^{(r)}: \mathcal{V} \mathcal{B}_{m} \rightarrow \mathcal{F M}$ (see the Introduction) is given by $V=T_{0}^{(r)} \mathbb{R}^{m}=$ $\left(J_{0}^{r}\left(\mathbb{R}^{m}, \mathbb{R}\right)_{0}\right)^{*}, H: G_{m}^{r} \rightarrow \mathrm{GL}\left(T_{0}^{(r)} \mathbb{R}^{m}\right),\left\langle H(\xi)(\omega), j_{0}^{r} \gamma\right\rangle=\left\langle\omega, j_{0}^{r}(\gamma \circ \varphi)\right\rangle$, $t: \mathcal{D}_{m}^{r} \rightarrow \operatorname{gl}\left(T_{0}^{(r)} \mathbb{R}^{m}\right),\left\langle t(\varrho)(\omega), j_{0}^{r} \gamma\right\rangle=\left\langle\omega, j_{0}^{r}(\eta \gamma)\right\rangle$ for $\omega \in\left(J_{0}^{r}\left(\mathbb{R}^{m}, \mathbb{R}\right)_{0}\right)^{*}$, $\xi=j_{0}^{r} \varphi \in G_{m}^{r}, j_{0}^{r} \gamma \in J_{0}^{r}\left(\mathbb{R}^{m}, \mathbb{R}\right)_{0}, \varrho=j_{0}^{r} \eta \in \mathcal{D}_{m}^{r}$.

Let $F: \mathcal{V B}_{m} \rightarrow \mathcal{F M}$ be a fiber product preserving gauge bundle functor of order $r$. Define a new fiber product preserving gauge bundle functor $\left(F\left(^{*}\right)\right)^{*}: \mathcal{V} \mathcal{B}_{m} \rightarrow \mathcal{F} \mathcal{M}$ by $E \mapsto\left(F E^{*}\right)^{*}$ and $f \mapsto\left(F f^{*}\right)^{*}$, where ()$^{*}$ denotes the dualization of $\mathcal{V} \mathcal{B}_{m}$-objects and $\mathcal{V} \mathcal{B}_{m}$-maps.

FACT 7. Let $(V, H, t)$ be an admissible triple of order $r$ corresponding to a fiber product preserving gauge bundle functor $F: \mathcal{V} \mathcal{B}_{m} \rightarrow \mathcal{F M}$ of order $r$. The admissible triple corresponding to $\left(F\left({ }^{*}\right)\right)^{*}: \mathcal{V} \mathcal{B}_{m} \rightarrow \mathcal{F} \mathcal{M}$ is $\left(V^{*}, H^{*}, t^{*}\right)$, where $H^{*}(\xi)=\left(H\left(\xi^{-1}\right)\right)^{*}$ for $\xi \in G_{m}^{r}$ and $t^{*}(\eta)=(t(\eta))^{*}$ for $\eta \in \mathcal{D}_{m}^{r}$.

Let $F_{1}, F_{2}: \mathcal{V} \mathcal{B}_{m} \rightarrow \mathcal{F M}$ be fiber product preserving gauge bundle functors of order $r$. Define $F_{1} \times_{B_{\mathcal{V} \mathcal{B}_{m}}} F_{2}: \mathcal{V} \mathcal{B}_{m} \rightarrow \mathcal{F M}$ by $\left(F_{1} \times_{B_{\mathcal{V} \mathcal{B}_{m}}} F_{2}\right)(E)=$ $F_{1} E \times_{M} F_{2} E$ for any $\mathcal{V} \mathcal{B}_{m}$-object $E \rightarrow M$ and $\left(F_{1} \times_{B_{\mathcal{V} \mathcal{B}_{m}}} F_{2}\right)(f)\left(v_{1}, v_{2}\right)=$ 
$\left(F_{1} f\left(v_{1}\right), F_{2} f\left(v_{2}\right)\right)$ for any $\mathcal{V} \mathcal{B}_{m}$-map $f: E \rightarrow G$ and any $\left(v_{1}, v_{2}\right) \in F_{1} E \times_{M}$ $F_{2} E$. Then $F_{1} \times_{B_{\mathcal{V} \mathcal{B}_{m}}} F_{2}: \mathcal{V} \mathcal{B}_{m} \rightarrow \mathcal{F} \mathcal{M}$ is again a fiber product preserving gauge bundle functor of order $r$. Let $\left(V^{F_{1}}, H^{F_{1}}, t^{F_{1}}\right),\left(V^{F_{2}}, H^{F_{2}}, t^{F_{2}}\right)$ and $\left(V^{F_{1} \times_{B_{\mathcal{V}} \mathcal{B}_{m}} F_{2}}, H^{F_{1} \times_{B_{\mathcal{V} \mathcal{B}_{m}}} F_{2}}, t^{F_{1} \times_{B_{\mathcal{V} \mathcal{B}_{m}}} F_{2}}\right)$ be the admissible triples corresponding to $F_{1}, F_{2}$ and $F_{1} \times_{B_{\mathcal{V} \mathcal{B}_{m}}} F_{2}$ respectively. We have a new admissible triple $\left(V^{F_{1}} \oplus V^{F_{2}}, H^{F_{1}} \oplus H^{F_{2}}, t^{F_{1}} \oplus t^{F_{2}}\right)$ of order $r$ and dimension $m$ such that $\left(H^{F_{1}} \oplus H^{F_{2}}\right)(\xi)=H^{F_{1}}(\xi) \oplus H^{F_{2}}(\xi): V^{F_{1}} \oplus V^{F_{2}} \rightarrow V^{F_{1}} \oplus V^{F_{2}}$ for any $\xi \in G_{m}^{r}$ and $\left(t^{F_{1}} \oplus t^{F_{2}}\right)(\eta)=t^{F_{1}}(\eta) \oplus t^{F_{2}}(\eta): V^{F_{1}} \oplus V^{F_{2}} \rightarrow V^{F_{1}} \oplus V^{F_{2}}$ for any $\eta \in \mathcal{D}_{m}^{r}$.

FACT 8. We have $\left(V^{F_{1} \times B_{\mathcal{V} \mathcal{B}_{m}} F_{2}}, H^{F_{1} \times_{B_{\mathcal{V} \mathcal{B}_{m}}} F_{2}}, t^{F_{1} \times_{B_{\mathcal{V} \mathcal{B}_{m}}} F_{2}}\right)=$ $\left(V^{F_{1}} \oplus V^{F_{2}}, H^{F_{1}} \oplus H^{F_{2}}, t^{F_{1}} \oplus t^{F_{2}}\right)$.

Let $F_{1}, F_{2}: \mathcal{V B}_{m} \rightarrow \mathcal{F} \mathcal{M}$ be fiber product preserving gauge bundle functors of orders $r_{1}$ and $r_{2}$ (respectively). Then $F_{1}$ and $F_{2}$ are of order $r=r_{1}+r_{2}$. Since $F_{2}$ has values in $\mathcal{V B}_{m}$ (see Lemma 1 ), we have the composition $F_{1} \circ F_{2}: \mathcal{V} \mathcal{B}_{m} \rightarrow \mathcal{F M}$ which is a fiber product preserving gauge bundle functor of order $r$. Let $\left(V^{F_{1}}, H^{F_{1}}, t^{F_{1}}\right),\left(V^{F_{2}}, H^{F_{2}}, t^{F_{2}}\right)$ and $\left(V^{F_{1} \circ F_{2}}, H^{F_{1} \circ F_{2}}, t^{F_{1} \circ F_{2}}\right)$ be the admissible triples of order $r$ and dimension $m$ corresponding to $F_{1}, F_{2}$ and $F_{1} \circ F_{2}$ respectively. By tensoring over $\mathbb{R}$ we have the admissible triple $\left(V^{F_{1}} \otimes V^{F_{2}}, H^{F_{1}} \otimes H^{F_{2}}, t^{F_{1}} \otimes t^{F_{2}}\right)$ of order $r$ and dimension $m$, where (of course) $\left(H^{F_{1}} \otimes H^{F_{2}}\right)(\xi)=H^{F_{1}}(\xi) \otimes H^{F_{2}}(\xi)$ : $V^{F_{1}} \otimes V^{F_{2}} \rightarrow V^{F_{1}} \otimes V^{F_{2}}$ for any $\xi \in G_{m}^{r}$ and $\left(t^{F_{1}} \otimes t^{F_{2}}\right)(\eta)=t^{F_{1}}(\eta) \otimes t^{F_{2}}(\eta)$ : $V^{F_{1}} \otimes V^{F_{2}} \rightarrow V^{F_{1}} \otimes V^{F_{2}}$ for any $\eta \in \mathcal{D}_{m}^{r}$.

Open Problem. Express $\left(V^{F_{1} \circ F_{2}}, H^{F_{1} \circ F_{2}}, t^{F_{1} \circ F_{2}}\right)$ by $\left(V^{F_{1}}, H^{F_{1}}, t^{F_{1}}\right)$ and $\left(V^{F_{2}}, H^{F_{2}}, t^{F_{2}}\right)$. Is $\left(V^{F_{1} \circ F_{2}}, H^{F_{1} \circ F_{2}}, t^{F_{1} \circ F_{2}}\right)$ canonically isomorphic to $\left(V^{F_{1}} \otimes V^{F_{2}}, H^{F_{1}} \otimes H^{F_{2}}, t^{F_{1}} \otimes t^{F_{2}}\right)$ ? In my opinion, it is not. Otherwise, there is a natural exchanging automorphism of $J^{1} \circ J^{1}: \mathcal{V} \mathcal{B}_{m} \rightarrow \mathcal{F} \mathcal{M}$. But this is rather impossible because an exchanging automorphism of $J^{1} \circ J^{1}$ : $\mathcal{F M}_{m} \rightarrow \mathcal{F} \mathcal{M}$ (over $\mathcal{F} \mathcal{M}_{m}$ ) does not exist (see [5], [7]).

\section{Classification of fiber product preserving gauge bundle func-} tors on $\mathcal{V B}_{m}$ of order $r$ in terms of admissible triples of order $r$ and dimension $m$. The following classification proposition shows that any fiber product preserving gauge bundle functor on $\mathcal{V B}_{m}$ of order $r$ is equivalent to some fiber product preserving gauge bundle functor as in Example 1.

Proposition 1. Let $F: \mathcal{V B}_{m} \rightarrow \mathcal{F} \mathcal{M}$ be a fiber product preserving gauge bundle functor of order $r$. Let $\left(V^{F}, H^{F}, t^{F}\right)$ be the admissible triple (of order $r$ and dimension $m$ ) corresponding to $F$. Then we have a natural equivalence $\Theta^{F}: F \cong T^{\left(V^{F}, H^{F}, t^{F}\right)}$.

Proof. Let $p: E \rightarrow M$ be a $\mathcal{V} \mathcal{B}_{m}$-object. We construct canonically a diffeomorphism $\Theta^{F}: F E \rightarrow T^{\left(V^{F}, H^{F}, t^{F}\right)} E$ as follows. Given a point $y \in$ 
$F_{x} E, x \in M$, we define $\Theta^{F}(y): J^{r} \mathcal{C}_{x}^{\infty, f l}(E) \rightarrow \widetilde{V}_{x}^{F} M$ by

$$
\Theta^{F}(y)(\xi)=F_{x}(f)(y) \in F_{x}(M \times \mathbb{R}) \cong \widetilde{V}_{x}^{F} M, \quad \xi=j_{x}^{r} f \in J^{r} \mathcal{C}^{\infty, f l}(E),
$$

where a fiber linear map $f: E \rightarrow \mathbb{R}$ is (in an obvious way) considered as the $\mathcal{V} \mathcal{B}_{m}$-map $f: E \rightarrow M \times \mathbb{R}$ covering the identity of $M$ and where the identification $F_{x}(M \times \mathbb{R}) \cong \widetilde{V}_{x}^{F} M$ is given by $F_{x}(M \times \mathbb{R}) \ni F\left(\varphi \times \operatorname{id}_{\mathbb{R}}\right)(v) \cong$ $\left\langle j_{0}^{r} \varphi, v\right\rangle \in \widetilde{V}_{x}^{F} M$ for $v \in V^{F}=F_{0}\left(\mathbb{R}^{m} \times \mathbb{R}\right)$, where $\varphi: \mathbb{R}^{m} \rightarrow M$ is an embedding with $\varphi(0)=x$. Then $\Theta^{F}(y)$ is well defined because $F$ is of order $r$. Recalling the definition of $\left(V^{F}, H^{F}, t^{F}\right)$ (see Example 2) and using the functoriality of $F$ one can verify in a standard (but long) way that $\Theta^{F}(y)$ is a module homomorphism over $t_{x}^{F}: J_{x}^{r}(M, \mathbb{R}) \rightarrow \operatorname{gl}\left(\widetilde{V}_{x}^{F} M\right)$, i.e. $\Theta^{F}(y) \in T_{x}^{\left(V^{F}, H^{F}, t^{F}\right)} E$.

It remains to show that $\Theta^{F}: F E \rightarrow T^{\left(V^{F}, H^{F}, t^{F}\right)} E$ is a diffeomorphism.

Because $\Theta^{F}: F \rightarrow T^{\left(V^{F}, H^{F}, t^{F}\right)}$ is natural with respect to $\mathcal{V} \mathcal{B}_{m}$-maps, and $F$ and $T^{\left(V^{F}, H^{F}, t^{F}\right)}$ preserve fiber products, and $E$ is locally a (multi) fiber product of $\mathbb{R}^{m} \times \mathbb{R}$, we may assume that $E=\mathbb{R}^{m} \times \mathbb{R}$, the trivial vector bundle over $\mathbb{R}^{m}$ with fiber $\mathbb{R}$. But for $E=\mathbb{R}^{m} \times \mathbb{R}$ the transformation $\Theta^{F}$ is the composition $F\left(\mathbb{R}^{m} \times \mathbb{R}\right) \cong \mathbb{R}^{m} \times V^{F} \cong T^{\left(V^{F}, H^{F}, t^{F}\right)}\left(\mathbb{R}^{m} \times \mathbb{R}\right)$, where the first identification is given by $F_{x}\left(\mathbb{R}^{m} \times \mathbb{R}\right) \ni v=\left(x, F\left(\tau_{-x} \times \mathrm{id}_{\mathbb{R}}\right)(v)\right) \in$ $\{x\} \times V^{F}, x \in \mathbb{R}^{m}$, and the second trivialization is induced (see Example 1) by the obvious trivialization of $\mathbb{R}^{m} \times \mathbb{R}$.

\section{Classification of admissible triples of order $r$ and dimension} $m$ in terms of fiber product preserving gauge bundle functors on $\mathcal{V} \mathcal{B}_{m}$ of order $r$. The following classification proposition shows that any admissible triple of order $r$ and dimension $m$ is isomorphic to some admissible triple as in Example 2.

Proposition 2. Let $(V, H, t)$ be an admissible triple of order $r$ and dimension $m$. Let $F=T^{(V, H, t)}$. Then we have an isomorphism $\mathcal{O}^{(V, H, t)}$ : $(V, H, t) \cong\left(V^{F}, H^{F}, t^{F}\right)$ of admissible triples.

We recall that a morphism $\left(V_{1}, H_{1}, t_{1}\right) \rightarrow\left(V_{2}, H_{2}, t_{2}\right)$ of admissible triples is a linear map $\mathcal{O}: V_{1} \rightarrow V_{2}$ such that $H_{2}(\xi) \circ \mathcal{O}=\mathcal{O} \circ H_{1}(\xi)$ for any $\xi \in G_{m}^{r}$ and $t_{2}(\eta) \circ \mathcal{O}=\mathcal{O} \circ t_{1}(\eta)$ for any $\eta \in \mathcal{D}_{m}^{r}$.

Proof. The composition $\mathcal{O}^{(V, H, t)}: V \rightarrow V^{F}=\operatorname{Hom}_{t_{0}}\left(J^{r} \mathcal{C}_{0}^{\infty, f l}\left(\mathbb{R}^{m} \times \mathbb{R}\right)\right.$, $\left.\widetilde{V}_{0} \mathbb{R}^{m}\right) \cong\{0\} \times V$ of $\mathcal{O}^{(V, H, t)}$ with the isomorphism induced (see Example 1) by the usual trivialization of $\mathbb{R}^{m} \times \mathbb{R}$ is the (almost) identity map. One can show in a standard (but long) way that $\mathcal{O}^{(V, H, t)}$ is a morphism $(V, H, t) \rightarrow$ $\left(V^{F}, H^{F}, t^{F}\right)$ of admissible triples. 
6. Natural transformations of fiber product preserving gauge bundle functors on $\mathcal{V} \mathcal{B}_{m}$ of order $r$ and induced morphisms between admissible triples. Let $F_{1}, F_{2}: \mathcal{V} \mathcal{B}_{m} \rightarrow \mathcal{F} \mathcal{M}$ be fiber product preserving gauge bundle functors of order $r$. Let $\left(V^{F_{1}}, H^{F_{1}}, t^{F_{1}}\right)$ and $\left(V^{F_{2}}, H^{F_{2}}, t^{F_{2}}\right)$ be the corresponding admissible triples of order $r$ and dimension $m$. Let $\mu: F_{1} \rightarrow F_{2}$ be a natural transformation.

EXAmple 3. Define $\nu^{\mu}: V^{F_{1}} \rightarrow V^{F_{2}}$ to be the restriction and corestriction of $\mu: F_{1}\left(\mathbb{R}^{m} \times \mathbb{R}\right) \rightarrow F_{2}\left(\mathbb{R}^{m} \times \mathbb{R}\right)$ to $V^{F_{1}}=\left(F_{1}\right)_{0}\left(\mathbb{R}^{m} \times \mathbb{R}\right)$ and $V^{F_{2}}=\left(F_{2}\right)_{0}\left(\mathbb{R}^{m} \times \mathbb{R}\right)$. Then $\nu^{\mu}:\left(V^{F_{1}}, H^{F_{1}}, t^{F_{1}}\right) \rightarrow\left(V^{F_{2}}, H^{F_{2}}, t^{F_{2}}\right)$ is a morphism of admissible triples. If $\mu$ is an isomorphism, then so is $\nu^{\mu}$.

Definition 4. We call $\nu^{\mu}$ the morphism corresponding to $\mu$.

7. Morphisms between admissible triples and induced natural transformations between fiber product preserving gauge bundle functors. Let $\left(V_{1}, H_{1}, t_{1}\right)$ and $\left(V_{2}, H_{2}, t_{2}\right)$ be admissible triples of order $r$ and dimension $m$. Let $\nu:\left(V_{1}, H_{1}, t_{1}\right) \rightarrow\left(V_{2}, H_{2}, t_{2}\right)$ be a morphism of admissible triples.

EXAmple 4. Given a $\mathcal{V} \mathcal{B}_{m}$-object $p: E \rightarrow M$ define a base preserving fibered map $\mu^{\nu}: T^{\left(V_{1}, H_{1}, t_{1}\right)} E \rightarrow T^{\left(V_{2}, H_{2}, t_{2}\right)} E$ as follows. Let $\Phi \in$ $T_{x}^{\left(V_{1}, H_{1}, t_{1}\right)} E=\operatorname{Hom}_{\left(t_{1}\right)_{x}}\left(J^{r} \mathcal{C}_{x}^{\infty, f l}(E),\left(\widetilde{V}_{1}\right)_{x} M\right), x \in M$. Put $\mu^{\nu}(\Phi)=\widetilde{\nu}_{x} \circ \Phi:$ $J^{r} \mathcal{C}_{x}^{\infty, f l}(E) \rightarrow\left(\widetilde{V}_{2}\right)_{x} M$, where $\widetilde{\nu}_{x}:\left(\widetilde{V}_{1}\right)_{x} M \rightarrow\left(\widetilde{V}_{2}\right)_{x} M, \widetilde{\nu}_{x}\left(\left\langle j_{x}^{r} \varphi, v\right\rangle\right)=$ $\left\langle j_{x}^{r} \varphi, \nu(v)\right\rangle$ for $v \in V_{1}$ and $\varphi: \mathbb{R}^{m} \rightarrow M$ is an embedding with $\varphi(0)=x$. We see that $\mu^{\nu}(\Phi) \in \operatorname{Hom}_{\left(t_{2}\right)_{x}}\left(J^{r} \mathcal{C}_{x}^{\infty, f l}(E),\left(\widetilde{V}_{2}\right)_{x} M\right)=T_{x}^{\left(V_{2}, H_{2}, t_{2}\right)} E$ and that $\mu^{\nu}: T^{\left(V_{1}, H_{1}, t_{1}\right)} \rightarrow T^{\left(V_{2}, H_{2}, t_{2}\right)}$ is a natural transformation. If $\nu$ is an isomorphism, then so is $\mu^{\nu}$.

DeFINITION 5. We call $\mu^{\nu}$ the natural transformation corresponding to $\nu$.

8. Object classification theorem. The first main result in this paper is the following theorem.

Theorem 1. The correspondence $F \mapsto\left(V^{F}, H^{F}, t^{F}\right)$ induces a bijective correspondence between the equivalence classes of fiber product preserving gauge bundle functors $F$ on $\mathcal{V B}_{m}$ of order $r$ and the equivalence classes of admissible triples $(V, H, t)$ of order $r$ and dimension $m$. The inverse correspondence is induced by $(V, H, t) \mapsto T^{(V, H, t)}$.

Proof. The correspondence $[F] \mapsto\left[\left(V^{F}, H^{F}, t^{F}\right)\right]$ is well defined, for if $\mu: F_{1} \rightarrow F_{2}$ is an isomorphism, then so is $\nu^{\mu}:\left(V^{F_{1}}, H^{F_{1}}, t^{F_{1}}\right) \rightarrow$ $\left(V^{F_{2}}, H^{F_{2}}, t^{F_{2}}\right)$. 
The correspondence $[(V, H, t)] \mapsto\left[T^{(V, H, t)}\right]$ is well defined, for if $\nu$ : $\left(V_{1}, H_{1}, t_{1}\right) \rightarrow\left(V_{2}, H_{2}, t_{2}\right)$ is an isomorphism, then so is $\mu^{\nu}: T^{\left(V_{1}, H_{1}, t_{1}\right)} \rightarrow$ $T^{\left(V_{2}, H_{2}, t_{2}\right)}$.

From Proposition 1 it follows that $[F]=\left[T^{\left(V^{F}, H^{F}, t^{F}\right)}\right]$. From Proposition 2 it follows that $[(V, H, t)]=\left[\left(V^{F}, H^{F}, t^{F}\right)\right]$ if $F=T^{(V, H, t)}$.

9. Morphism classification theorem. Let $F_{1}$ and $F_{2}$ be fiber product preserving gauge bundle functors on $\mathcal{V B}_{m}$ of order $r$. Let $\left(V^{F_{1}}, H^{F_{1}}, t^{F_{1}}\right)$ and $\left(V^{F_{2}}, H^{F_{2}}, t^{F_{2}}\right)$ be the corresponding admissible triples of order $r$ and dimension $m$.

LEMMA 2. Let $\nu:\left(V^{F_{1}}, H^{F_{1}}, t^{F_{1}}\right) \rightarrow\left(V^{F_{2}}, H^{F_{2}}, t^{F_{2}}\right)$ be a morphism of admissible triples. Let $\mu^{[\nu]}: F_{1} \rightarrow F_{2}$ be a natural transformation given by the composition

$$
F_{1} \stackrel{\Theta^{F_{1}}}{\longrightarrow} T^{\left(V^{F_{1}}, H^{F_{1}}, t^{F_{1}}\right)} \stackrel{\mu^{\nu}}{\longrightarrow} T^{\left(V^{F_{2}}, H^{F_{2}}, t^{F_{2}}\right)} \stackrel{\left(\Theta^{F_{2}}\right)^{-1}}{\longrightarrow} F_{2},
$$

where $\Theta^{F}$ is as in Proposition 1 and $\mu^{\nu}$ is described in Example 4. Then $\mu=\mu^{[\nu]}$ is the unique natural transformation $F_{1} \rightarrow F_{2}$ such that $\nu^{\mu}=\nu$, where $\nu^{\mu}$ is as in Example 3.

Proof. Suppose $\bar{\mu}: F_{1} \rightarrow F_{2}$ is another natural transformation such that $\nu^{\bar{\mu}}=\nu$. Then $\bar{\mu}$ coincides with $\mu$ on the vector bundle $\mathbb{R}^{m} \times \mathbb{R}$. Hence $\bar{\mu}=\mu$ by the same argument as in the proof of Proposition 1.

Now, the following second main result of this paper is clear.

THEOREM 2. Let $F_{1}$ and $F_{2}$ be two fiber product preserving gauge bundle functors on $\mathcal{V} \mathcal{B}_{m}$ of order $r$. The correspondence $\mu \mapsto \nu^{\mu}$ is a bijection between natural transformations $F_{1} \rightarrow F_{2}$ and morphisms $\left(V^{F_{1}}, H^{F_{1}}, t^{F_{1}}\right) \rightarrow$ $\left(V^{F_{2}}, H^{F_{2}}, t^{F_{2}}\right)$ between corresponding admissible triples. The inverse correspondence is $\nu \mapsto \mu^{[\nu]}$.

\section{Finite order theorem}

THEOREM 3. Any fiber product preserving gauge bundle functor $F: \mathcal{V B}_{m}$ $\rightarrow \mathcal{F M}$ is of finite order.

Proof. Define $A^{F}: \mathcal{C}^{\infty, f l}\left(\mathbb{R}^{m} \times \mathbb{R}\right) \rightarrow \mathcal{C}^{\infty}\left(F\left(\mathbb{R}^{m} \times \mathbb{R}\right), F\left(\mathbb{R}^{m} \times \mathbb{R}\right)\right)$ by $A^{F}(f)=F f$, where a fiber linear map $f: \mathbb{R}^{m} \times \mathbb{R} \rightarrow \mathbb{R}$ is considered as a base preserving $\mathcal{V} \mathcal{B}_{m}$-map $\mathbb{R}^{m} \times \mathbb{R} \rightarrow \mathbb{R}^{m} \times \mathbb{R}$ in an obvious way. Clearly, $A^{F}$ is $\pi$-local, where $\pi: F\left(\mathbb{R}^{m} \times \mathbb{R}\right) \rightarrow \mathbb{R}^{m}$ is the projection. The zero map $O \in \mathcal{C}^{\infty, f l}\left(\mathbb{R}^{m} \times \mathbb{R}\right)$ is invariant with respect to the translations of $\mathbb{R}^{m}$. Moreover, given $f \in \mathcal{C}^{\infty, f l}\left(\mathbb{R}^{m} \times \mathbb{R}\right), v \in F_{0}\left(\mathbb{R}^{m} \times \mathbb{R}\right)$, a neighborhood $W$ of $j_{0}^{\infty}(0)$ and a neighborhood $U$ of $0 \in F_{0}\left(\mathbb{R}^{m} \times \mathbb{R}\right)$, there is $t \in \mathbb{R}_{+}$such that $j_{0}^{r}\left(f \circ \lambda_{t}\right) \in W$ and $F \lambda_{t}(v) \in U$, where $\lambda_{t}$ is the fiber homothety by $t$. Then 
by the non-linear Peetre theorem [5] we deduce that the operator $A^{F}$ is of finite order $r_{1}$. In particular, $F_{0} f$ depends on $j_{0}^{r_{1}} f$ only.

We have the bundle functor $G^{F}: \mathcal{M} f_{m} \rightarrow \mathcal{F M}$ such that $G^{F} M=$ $F(M \times \mathbb{R})$ for any $m$-manifold $M$ and $G^{F} \varphi=F\left(\varphi \times \operatorname{id}_{\mathbb{R}}\right): G^{F} M \rightarrow G^{F} N$ for any embedding $\varphi: M \rightarrow N$ between $m$-manifolds. By the Palais-Terng theorem (see [5]), $G^{F}$ has finite order $r_{2}$.

We prove that $F$ is of order $r=\max \left(r_{1}, r_{2}\right)$. We consider a $\mathcal{V} \mathcal{B}_{m}$-map $f: E_{1} \rightarrow E_{2}$ and a point $x \in M_{1}$. It remains to show that $F_{x} f$ depends on $j_{x}^{r} f$.

Using $\mathcal{V} \mathcal{B}_{m}$-trivializations we can assume that $E_{1}=\mathbb{R}^{m} \times \mathbb{R}^{n}, E_{2}=$ $\mathbb{R}^{m} \times \mathbb{R}^{q}$ and $x=0 \in \mathbb{R}^{m}$. Since $F$ preserves fiber products, we can assume that $q=1$. For the same reason, we can assume that $n=1$. (Apply the fact that the map $I: \times_{M}^{n} F\left(\mathbb{R}^{m} \times \mathbb{R}\right) \rightarrow F\left(\mathbb{R}^{m} \times \mathbb{R}^{n}\right)$ inverse to the fiber product identification $F\left(\mathbb{R}^{m} \times \mathbb{R}^{n}\right) \rightarrow \times_{M}^{n} F\left(\mathbb{R}^{m} \times \mathbb{R}\right)$ is given by $I\left(v_{1}, \ldots, v_{n}\right)=$ $\sum_{j} F\left(g^{j}\right)\left(v^{j}\right)$ for $v_{1}, \ldots, v_{n} \in F_{x}\left(\mathbb{R}^{m} \times \mathbb{R}\right), x \in \mathbb{R}^{m}, g^{j}: \mathbb{R}^{m} \times \mathbb{R} \rightarrow \mathbb{R}^{m} \times \mathbb{R}^{n}$, $g^{j}(x, y)=\left(x, e_{j} y\right), e_{j}=(0, \ldots, 0,1,0, \ldots, 0)(1$ in position $j), j=1, \ldots, n$, where the sum is the one in the vector bundle $F\left(\mathbb{R}^{m} \times \mathbb{R}^{n}\right)$; see Lemma 1.) Then we can write $f=\widetilde{f} \circ\left(\varphi \times \operatorname{id}_{\mathbb{R}}\right)$, where $\widetilde{f}: \mathbb{R}^{m} \times \mathbb{R} \rightarrow \mathbb{R}^{m} \times \mathbb{R}$ is a base preserving $\mathcal{V B}_{m}$-map and $\varphi: \mathbb{R}^{m} \rightarrow \mathbb{R}^{m}$ is a 0 -preserving embedding. Then $F_{0} f=F_{0} \widetilde{f} \circ G_{0}^{F} \varphi$ depends on $j_{0}^{r_{1}} \widetilde{f}$ and $j_{0}^{r_{2}} \varphi$ as we showed above.

11. Applications. We give some applications of the main results. We will use the Facts from Section 3.

Lemma 3. Let $a: \mathcal{D}_{m}^{r} \rightarrow \mathcal{D}_{m}^{r}$ be a $G_{m}^{r}$-equivariant linear map, where $\mathcal{D}_{m}^{r}$ is a $G_{m}^{r}$-space via $H_{m}^{r}$ as in Fact 1 . Then there exist unique $k, l \in \mathbb{R}$ such that $a=k \operatorname{id}_{\mathcal{D}_{m}^{r}}+l \varepsilon_{m}^{r}$, where $\operatorname{id}_{\mathcal{D}_{m}^{r}}$ is the identity map and $\varepsilon_{m}^{r}$ is the map as in Fact 2.

Proof. Below $\alpha, \beta \in(\mathbb{N} \cup\{0\})^{m}$ with $|\alpha| \leq r$ and $|\beta| \leq r$. We can write $a\left(j_{0}^{r} x^{\alpha}\right)=\sum_{\beta} a_{\beta}^{\alpha} j_{0}^{r} x^{\beta}$ for some unique real numbers $a_{\beta}^{\alpha}$. By the equivariance of $a$ with respect to $j_{0}^{r}\left(\tau^{1} x^{1}, \ldots, \tau^{m} x^{m}\right) \in G_{m}^{r}$ we deduce that $\tau^{\alpha} a_{\beta}^{\alpha}=\tau^{\beta} a_{\beta}^{\alpha}$ for any $\tau=\left(\tau^{1}, \ldots, \tau^{m}\right) \in \mathbb{R}_{+}^{m}$. Then $a\left(j_{0}^{r} x^{\alpha}\right)=a_{\alpha}^{\alpha} j_{0}^{r} x^{\alpha}$. If $\alpha \neq(0)$, then by the equivariance of $a$ with respect to $j_{0}^{r}\left(x^{1}+x^{\alpha}, x^{2}, \ldots, x^{m}\right) \in G_{m}^{r}$ we deduce that $a_{e_{1}}^{e_{1}} j_{0}^{r} x^{1}+a_{\alpha}^{\alpha} j_{0}^{r} x^{\alpha}=a\left(j_{0}^{r}\left(x^{1}+x^{\alpha}\right)\right)=a_{e_{1}}^{e_{1}}\left(j_{0}^{r} x^{1}+j_{0}^{r} x^{\alpha}\right)$, i.e. $a\left(j_{0}^{r} x^{\alpha}\right)=a_{e_{1}}^{e_{1}} j_{0}^{r} x^{\alpha}$. So, it remains to put $k=a_{e_{1}}^{e_{1}}$ and $l=a_{(0)}^{(0)}-a_{e_{1}}^{e_{1}}$.

Lemma 4. Suppose that $a: \mathcal{D}_{m}^{r} \rightarrow \mathcal{D}_{m}^{r}$ is a $G_{m}^{r}$-equivariant linear map such that $t_{m}^{r}(\eta) \circ a=a \circ t_{m}^{r}(\eta)$ for any $\eta=j_{0}^{r} \gamma \in \mathcal{D}_{m}^{r}$, where $t_{m}^{r}$ is as in Fact 1. Then there exists a unique $k \in \mathbb{R}$ such that $a=k \operatorname{id}_{\mathcal{D}_{m}^{r}}$.

Proof. By Lemma 3, $a=k \operatorname{id}_{\mathcal{D}_{m}^{r}}+l \varepsilon_{m}^{r}$. Since $a$ commutes with $t_{m}^{r}$, we easily obtain $l=0$. 
Lemma 5. The space of endomorphisms of $\left(V, H, \varepsilon^{V}\right)$ (see Fact 5$)$ is the space of $G_{m}^{r}$-equivariant linear endomorphisms of $V$, where $V$ is a $G_{m}^{r}$-space via $H$.

Proof. This is a simple observation.

Corollary 1. Any natural endomorphism $\mu: J^{r} \rightarrow J^{r}$ is $k \mathrm{id}_{J^{r}}$ for some uniquely determined real number $k$.

Proof. By Theorem 2 natural endomorphisms $J^{r} \rightarrow J^{r}$ are in bijection with endomorphisms of the admissible triple $\left(\mathcal{D}_{m}^{r}, H_{m}^{r}, t_{m}^{r}\right)$ corresponding to $J^{r}$ (see Fact 1). By Lemma 4 these endomorphisms are $k \operatorname{id}_{\mathcal{D}_{m}^{r}}$ for $k \in \mathbb{R}$.

COROLlary 2. Any natural endomorphism $\mu: J_{v}^{r} \rightarrow J_{v}^{r}$ is $k \mathrm{id}_{J_{v}^{r}}+l \mu_{m}^{r}$ for some uniquely determined real numbers $k, l$, where for any $\mathcal{V} \mathcal{B}_{m}$-object $p: E \rightarrow M$ we have $\mu_{m}^{r}: J_{v}^{r} E \rightarrow J_{v}^{r} E$ with $\mu\left(j_{x}^{r} \gamma\right)=j_{x}^{r}(\gamma(x))$ for $\gamma: M \rightarrow E_{x}, x \in M$, where $\gamma(x): M \rightarrow E_{x}$ is the constant map.

Proof. The proof is quite similar to that of Corollary 1. We use Theorem 2, Lemma 3 and Fact 2.

COROLlary 3. Any natural endomorphism $\mu: \widetilde{\widetilde{V}} E \rightarrow \widetilde{\widetilde{V}} E$ for any $\mathcal{V} \mathcal{B}_{m^{-}}$ object $p: E \rightarrow M$, where $\widetilde{\widetilde{V}}$ is as in Fact 5 , is $\mu=\operatorname{id}_{E} \otimes \bar{\mu}_{M}$ for some natural endomorphism $\bar{\mu}: \widetilde{V} M \rightarrow \widetilde{V} M$.

In particular, any natural endomorphism $\mu: V^{A} E=E \otimes A \rightarrow V^{A} E$, where $V^{A}$ is the vertical Weil gauge bundle functor corresponding to a Weil algebra $A$, is $\mu=\mathrm{id}_{E} \otimes B$ for some $B \in \operatorname{gl}(A)$.

Proof. The proof is quite similar to that of Corollary 1. We use Theorem 2, Lemma 5 and Fact 5 . That $V^{A} E=E \otimes A$ follows from the fact that the admissible triples corresponding to the functors are isomorphic.

\section{References}

[1] A. Cabras and I. Kolář, Prolongations of projectable tangent valued forms, Arch. Math. (Brno) 38 (2002), 243-257.

[2] M. Doupovec and I. Koláŕ, Iteration of fiber product preserving bundle functors, Monatsh. Math. 134 (2001), 39-50.

[3] I. Kolář, Bundle functors of the jet type, in: Differential Geometry and Applications (Brno, 1998), Masaryk Univ., Brno, 1999, 231-237.

[4] - On the geometry of fiber product preserving bundle functors, in: Differential Geometry and its Applications (Opava, 2001), Math. Publ. 3, Silesian Univ. Opava, Opava, 2001, 85-92.

[5] I. Kolář, P. Michor and J. Slovák, Natural Operations in Differential Geometry, Springer, 1993.

[6] I. Kolář and W. M. Mikulski, On the fiber product preserving bundle functors, Differential Geom. Appl. 11 (1999), 105-115. 
[7] I. Kolár and M. Modugno, Natural maps on the iterated jet prolongation of a fibered manifold, Ann. Mat. Pura Appl. (4) 158 (1991), 151-165.

[8] W. M. Mikulski, The natural operators lifting projectable vector fields to some fiber product preserving bundles, Ann. Polon. Math. 81 (2003), 261-271.

Institute of Mathematics

Jagiellonian University

Reymonta 4

30-059 Kraków, Poland

E-mail: mikulski@im.uj.edu.pl

Reçu par la Rédaction le 11.2.2003

Révisé le 2.4.2003 